\title{
CRYSTAL STRUCTURE DETERMINATION OF A N-ACYLHYDRAZONE DERIVATIVE: LASSBIO-1733
}

\author{
Costa, F.N. ${ }^{\text {, }}$; Silva, T.F. ${ }^{2}$; Sato, J.A.P. ; Barroso, R.C. ${ }^{3}$; Braz, D. ${ }^{4}$ ' Lima, L.M. ${ }^{2}$; \\ Barreiro, E.J. ${ }^{2}$ and Ferreira, F.F. ${ }^{1}$
}

${ }^{1}$ Center of Natural and Human Sciences, Federal University of ABC, Santo André, SP, Brazil

${ }^{2}$ LASSBio, Institute of Biomedical Sciences, Federal University of Rio de Janeiro, Rio de Janeiro, RJ, Brazil.

${ }^{3}$ Physics Institute, State University of Rio de Janeiro, Rio de Janeiro, RJ, Brazil.

${ }^{4}$ Program of Nuclear Engineering, Federal University of Rio de Janeiro, Rio de Janeiro, RJ, Brazil.

*fanny.costa@ufabc.edu.br

\begin{abstract}
The methodology of structure determination from X-ray diffraction data has been employed as a tool able to define the configurational and conformational aspects of the new bioactive compounds, which are directly related to the biological activity. In this work, X-ray powder diffraction (XRPD) was used to carry out the crystal structure determination of LASSBio-1733, which was initially obtained as part of a project of synthesis of novel anti-inflammatory and analgesic leads with a $N$-acylhydrazone scaffold. The measurements were performed at room temperature on a Stoe STADI-P powder diffractometer in transmission geometry by using a $\mathrm{CuK} \alpha_{1}(\lambda=1.54056 \AA)$ wavelength. LASSBio-1733 crystallizes in an orthorhombic crystal system, space group $P 2_{1} 2_{1} 2_{1}$, with unit-cell dimensions $a=$ 25.2049(13) $\AA, b=10.2952(6) \AA, c=5.2333(3) \AA, \alpha=\beta=\gamma=90^{\circ}, \mathrm{V}=1357.99(13) \AA^{3}$. The structure was energy-minimised with dispersion-corrected density functional theory (DFT-D). ${ }^{1}$ Additionally, other experimental techniques were employed in characterization of this compound.
\end{abstract}

Acknowledgements: FAPESP, CNPq, CAPES, INCT-Inofar and UFABC. Reference:

[1] Streek, J. v. d. \& Neumann, M. A. (2010). Acta Crystallogr. B 66, 544-558. 